\title{
Considérations sur la sédimentologie des estuaires
}

\author{
par H. Chamley \\ Géologie marine \\ Centre d'Océanographie, Centre Universitaire de Luminy \\ 13288 Marseille Cedex 2
}

\section{Introduction}

La définition morphologique de l'estuaire diffère selon les auteurs. Pritchard (in Lauff, 1967), avec maints chercheurs anglo-saxons, le considère comme « une aire côtière semi-fermée, en liaison libre avec la mer ouverte, et dans laquelle l'eau marine est diluée de façon mesurable dans l'eau issue du drainage continental $\gg$. Cette définition large permet d'inclure, au cas habituel des embouchures de fleuves, les fjords où s'ajoute une intervention glaciaire, les estuaires déterminés par l'existence d'une barre, et les estuaires d'origine tectonique.

En France, on définit l'estuaire de façon plus restrictive. comme la partie d'un fleuve où la marée se fait sentir (Guilcher, 1954; Derruau, 1967). Seules les mers à marées assez fortes sont bordées de véritables estuaires (Twenhofel, 1961). Les deux aspects de la marée, marée de salinité et marée dynamique, interviennent pour expliquer la nature et la répartition des sédiments. Le second affectant une aire fluviale bien plus étendue que le premier, nous considérons donc l'estuaire comme la partie d'un fleuve où agit la marée dynamique.

\section{Techniques d'étude}

Aux techniques habituellement mises en cuvre pour étudier les sédiments marins (dragages, carottages à tube étroit, mesure des teneurs en sable et calcaire, recherches faunistiques, minéralogiques, géochimiques...), on a dû adjoindre des techniques adaptées à la mobilité du milieu estuarien et à sa richesse en suspensions. La contribution de ces dernières années a été particulièrement fertile à cet égard, et témoigne du souci de disposer de résultats continus, en relation aussi étroite que possible avec les données hydrologiques.

Les suspensions d'estuaire sont suivies, dans leurs déplacements, à l'aide d'écho-sondeurs lorsqu'elles sont suffisamment denses (Allen, 1972) et, plus récemment, à l'aide de thermographies en infrarouges. S'y ajoutent des mesures de néphélémétrie en continu, de radio-activité naturelle ou artificielle (Giresse, 1969; Martin, 1971).

Les sédiments d'estuaire sont repérés à l'aide d'échosondeurs directs ou latéraux; la nature de l'écho enregistré permet, dans une certaine mesure, de savoir s'il s'agit de vase, de sable ou de roche consolidée. Les prélèvements ont lieu, de préférence, avec des carottiers de section large, parfois quadrangulaire (Reineck, in Lauff, 1967), dont l'utilisation ne perturbe pas les structures. Des plateformes spéciales, mobiles, ont été aménagées pour les carottages dans les vasières (Ottmann et al., 1970). Au laboratoire, l'emploi systématique de tests non destructifs sur les carottes, notamment la gammadensimétrie, la radiographie et les mesures acoustiques, permet d'apprécier la qualité du prélèvement, la nature et l'intérêt de l'échantillon. La rapidité de ces analyses autorise à multiplier l'échantillonnage. Enfin, les études géotechniques se développent et se diversifient (Ottmann et al., 1970).

\section{Nature des suspensions et sédiments}

A. - Les eaux estuariennes sont caractérisées par UNE GRANDE TURBIDITÉ, qui s'oppose souvent à celle des caux fluviales en amont, et des eaux marines en aval (ex. : Garonne : 0,1 à $1 \mathrm{~g} / 1$ à Libourne et à la pointe de Grave; plusieurs dizaines de $\mathrm{g} / \mathrm{l}$ dans la partie médiane de l'estuaire) 
Ces eaux, très chargées en suspensions, ont été appelées bouchon vaseux par Glangeaud (1938) en Gironde, où elles peuvent renfermer $4500000 t$ de matériel. Elles résultent de la reprise, par les ondes de marée, de sédiment fin déjà déposé ou en cours de dépôt.

Une autre formation turbide a été mise récemment en évidence dans la Gironde : il s'agit de la crème de vase ou "couche-crème» (fluid-mud des auteurs anglo-saxons), qui constitue une lentille de boue liquide sur le fond des chenaux de marée (Allen et al., 1970; Martin, 1971).

La composition de ces différents troubles n'a fait l'objet que de rares études (Germaneau, 1968 à 1971, en baie de Seine), mais elle paraît très voisine de celle des sédiments sous-jacents. Leur origine est mixte, à la fois fluviale et marine.

B. - LES SÉDIMENTS estuARIENS, bien que comportant localement des sables et des graviers, sont principalement formés de vase. Les constituants de la vase ont été classés selon leur nature ou selon leur origine. Bourcart (in Ottmann, 1965) reconnaît une phase inerte, qui comprend des particules minérales, surtout argileuses, ainsi que des débris organiques calcaires ou siliceux, et une phase «active» qui comprend l'eau d'imbibition, des sels et oxydes divers, et la matière organique. Germaneau (1968 à 1971) a récemment distingué, dans l'estuaire de la Seine, les éléments d'origine fluviale et marine, ceux qui proviennent de suspensions temporaires et semi-permanentes, ceux qui sont actuels et fossiles.

Les caractères physiques de la vase résident principalement dans sa teneur élevée en eau (souvent plus de $100 \%)$, sa viscosité, sa rigidité, sa compressibilité, sa thixotropie; ils paraissent déterminés par le mode d'agencement des particules qui, en emprisonnant des molécules d'eau, forment une structure en nid d'abeille.

Les propriétés géotechniques des vases d'estuaire sont encore mal connues. On sait que le taux de cisaillement est dépendant de la teneur en eau, en éléments fins et de la perméabilité; l'indice des vides est influencé par la teneur en matière organique (Ottmann et al., 1970). La teneur en eau augmente avec le pourcentage de particules fines, la salinité, la vitesse de sédimentation, la teneur en matière organique, l'abondance de minéraux argileux adsorbants comme la montmorillonite, la jeunesse du dépôt. La compaction de la vase varie en raison inverse de la température, et cela semble lié à l'activité biologique et microbiologique au sein du sédiment (McMaster, in Lauff, 1967).

La minéralogie des argiles a fait l'objet de nombreuses études, car les estuaires, comme les deltas, marquent le passage d'un milieu pauvre en ions, représenté par le fleuve, à un milieu plus riche, représenté par la mer : on peut s'attendre à ce que des modifications affectent les minéraux argileux détritiques, plus ou moins dégradés au cours de l'altération pédologique. De fait, des transformations de nature diverse, traduisant souvent une régularisation des réseaux argileux, ont été évoquées (in Millot, 1964), et le cas de la Gironde en constitue un exemple récent (Latouche, 1971). Mais la part est difficile à faire entre ce qui revient à la sédimentation différentielle (Whitehouse et al., in Millot, 1964) et aux transformations cristallographiques. Il semble bien que, dans la majorité des cas, le passage au milieu marin affecte peu les argiles héritées, puisqu'elles conservent fidèlement dans les dépôts marins le façonnement climatique qu'elles ont acquis sur le continent, avant leur érosion (Chamley, 1971).

L'étude géochimique des vases estuariennes s'oriente actuellement dans des voies variées. Un «faciès géochimique » est reconnu par Ottmann et al. (1970) dans l'estuaire de la Loire : il est caractérisé par davantage de $\mathrm{Si}$, moins de $\mathrm{Fe}$ et $\mathrm{Mg}$ que les faciès continental, lagunaire ou marin. Dans les vases bordières, les eaux interstitielles conservent parfois la salinité d'origine, qui permet alors de reconstituer les cycles de marée et les conditions de dépôt anciens. Les relations entre les eaux libres et les sédiments sont souvent évoquées (in Lecolle, 1972, et Monnet, 1972). Ainsi Martin (1971), en Gironde, montre la mise en solution des oligoéléments liés aux suspensions dans l'estuaire, puis leur fixation par les complexes organométalliques des sédiments près de l'embouchure. Monnet (1972) met en évidence l'importance des phénomènes mécaniques de mélange et d'adsorption par les gels, dans la répartition des éléments majeurs et en traces du Bandama (Côte d'Ivoire).

\section{Répartition des suspensions et sédiments}

A. - LE «BOUCHON VASEuX » semble exister dans la plupart des estuaires. Il est mobile et se déplace dans l'axe de l'estuaire avec la marée, dans le même sens qu'elle. Il se situe davantage vers l'amont en période d'étiage, davantage vers l'embouchure en période de crue. Sa position est indépendante de la salinité dans l'estuaire, car il ne résulte pas d'un processus de floculation et n'est conditionné que par la marée dynamique (Martin, 1971; Postma, in Lauff, 1967). Aux mouvements longitudinaux s'ajoutent des mouvements latéraux, qui sont à l'origine du dépôt de vase sur les bordures de l'estuaire.

La position des lentilles de crème de vase en Gironde (Allen, 1972) obéit à un cycle saisonnier : la crème se forme en amont de l'estuaire lors de l'étiage, et en aval lors des crues. Elle semble résulter du remplissage d'une "lentille d'eau immobile» (décrite pour la première fois par Berthois en 1955 dans la Loire) par des matériaux provenant du bouchon vaseux. Elle est redispersée dans le bouchon vaseux lors des marées de vives eaux, et se forme à nouveau lors des marées de moyennes et mortes eaux. La «couche-crème» paraît déterminée par la mise en agrégats d'argile dans des conditions de courants, de salinité et de topographie assez strictes (Martin, 1971).

B. - LA RÉPARTITION DES SÉDIMENTS dans l'estuaire peut être schématisée de la manière suivante (Ottmann, 1965) :

- Sens longitudinal, d'amont en aval :

- Zone fluviale : sable et graviers continentaux.

- Zone estuarienne stricte : vase issue de troubles.

- Zone de l'embouchure : bancs de sable marin, qui forment une «barre» plus ou moins marquée.

- Zone du large: vase fine décantée. 
- Sens transversal :

- Berges et bancs latéraux : vase, formant sur les bordures le shorre, qui tend à être colonisé par une végétation halophile, et dans la zone intertidale la slikke (= mud flats).

- Zone médiane : mélange complexe de bancs de vase et de chenaux plus ou moins sableux, voire graveleux.

La disposition des bancs de vase est souvent lenticulaire. Les forces hydrodynamiques en jeu se traduisent par des stratifications entrecroisées, des ripple marks et, dans les chenaux, des dunes hydrauliques très mobiles. Les vasières de la slikke sont parcourues par des micro-réseaux hydrographiques, où circulent à marée basse les eaux issues du flot et des nappes souterraines.

\section{Mise en place des sédiments}

A. - Le dépot du sable et des galets, qui sont charriés sur le fond, s'effectue lorsque la compétence des eaux diminue et ne permet plus de déplacement par traction ni par saltation. Ce dépôt a lieu principalement dans la zone amont de l'estuaire (diminution de la granularité vers l'aval), dans les chenaux et le long des îles, ainsi qu'à proximité de l'embouchure. Les grains se répartissent dans les différentes zones en fonction de leur taille, de leur densité et de leur forme.

B. - LE DÉPOT DE LA VASE, qui s'effectue surtout à partir de suspensions, pose un problème complexe, dans le milieu agité que représente l'estuaire.

Le mécanisme habituellement invoqué est celui de la floculation des argiles, qui sont des composants essentiels de la vase : les minéraux argileux, qui présentent en eau douce des forces de répulsion par suite d'un excès de charges négatives à leur périphérie, s'agrégeraient lorsqu'ils parviennent au contact des eaux marines relativement riches en cations. Ce mécanisme a été clémontré expérimentalement. Mais les conditions de son fonctionnement sont moins claires dans la nature, et il est certain que la floculation ne peut constituer le processus essentiel du dépôt de la vase pour de multiples raisons. Tout d'abord, floculation ne signifie pas sédimentation, et rien n'indique que de petits agrégats d'argile, de 10 à 20 microns de diamètre et gorgés d'eau, aient une masse suffisante pour se déposer (Chamley, 1971). Par ailleurs, la floculation n'est pas un phénomène aussi généralisé, ou du moins aussi massif, que l'on pense, car les conditions de sa manifestation sont variables : ces conditions semblent meilleures lorsque la densité de l'eau est faible, la température élevée, les eaux calmes, la charge ionique élevée; mais elles varient localement avec le $\mathrm{pH}$, la salinité, la nature des cations dissous, et parfois de manière encore inexpliquée. De plus, une floculation différentielle affecte les minéraux argileux considérés isolément (Whitehouse et al., in Millot, 1964; Postma, in Lauff, 1967); les mélanges de minéraux, qui constituent la règle dans le milieu naturel, posent des problèmes encore plus complexes. Enfin, des expériences ont montré que le milieu estuarien était, en fait, souvent peu propice à la floculation (Muller, in Van Straaten, 1964): les caux douces et marines dêterminent une floculation des argiles par adsorption de $\mathrm{Ca}$ et $\mathrm{Na}$ respectivement, ce qui donne des sédiments peu lités et très perméables; au contraire, les eaux saumâtres, dans lesquelles les argiles adsorbent préférentiellement $\mathrm{Mg}$, sont affectées par une certaine dispersion, qui conduit à un dépôt lent de particules stratifiées et compactées.

D'autres mécanismes interviennent donc dans le dépôt des vases d'estuaires :

- L'agglomération, par effet électrostatique, de particules en suspensions autour de débris grossiers, détermine des flocons (de 1 à $10 \mathrm{~mm}$ de longueur), qui peuvent se déposer plus facilement que les flocules.

- Au début du jusant, lorsque l'eau se retire des vasières de bordures, les particules se «collent» au sédiment qui découvre peu à peu, à la manière de la mousse sur les parois d'une baignoire qui se vide. C'est le phénomène d'accrétion (Francis-Bœuf, in Bourcart, 1958) ou de colmatage (Glangeaud, 1938), et les particules fines ainsi déposées, sur des surfaces d'inclinaisons variées, sont pratiquement impossibles à redisperser par les agents naturels communs. L'accrétion est favorisée par un «effet de retard » (lag effect de Postma, in Lauff, 1967), les particules fines ne se déposant qu'après l'amortissement complet du flot.

- Au niveau des chenaux de marée, là où la vase ne découvre pas à marée basse, on invoque un dépôt par décantation, qui a été décrit par Glangeaud (1938) à propos de la Gironde : de part et d'autre du courant de surface de jusant, à mi-hauteur dans la masse de l'eau, s'établissent des courants tourbillonnaires violents, entre lesquels existent des zones de calme où s'effectue le dépôt. Il en résulte des bancs vaseux médians dans le chenal, qui provoquent une division latérale du mouvement. Le mécanisme se poursuit jusqu'à ce qu'intervienne un changement du cours des eaux.

D'une façon générale, la décantation des suspensions paraît constituer un phénomène répandu, favorisé par des eaux calmes (étales de marée) et denses. Notons que la mobilité du bouchon vaseux explique la distribution généralisée des vases dans les différentes parties de l'estuaire.

\section{Origine et devenir des sédiments estuariens}

A. - La provenance des dépots d'estuaires peut être établie grâce à l'utilisation de «marqueurs» significatifs : tests d'organismes marins ou dulcaquicoles, pollen, minéraux sableux lourds ou argileux d'origine connue, carbone 13 de la matière organique continentale... Une telle démarche permet de faire aboutir la controverse, đéjà ancienne, sur l'origine fluviale ou marine des dépôts d'estuaires, et de montrer que différentes situations existent en fait :

- Sédiments d'origine marine : c'est le cas des estuaires de la mer du Nord (mer de Wadden, Ems) et du golfe normano-breton, où le marnage est élevé et le débit des fleuves faible. L'estuaire se comporte comme une baie 
marine, dont la zone d'équilibre sédimentaire se trouve à l'intérieur ou à l'extérieur de la ria, selon la position du niveau des basses mers (Berthois, 1970). Une situation voisine s'observe lorsque les apports proviennent, non pas du large ou d'un transiert littoral, mais de lagunes et marais maritimes situés près de l'embouchure (fleuve Kapatchez en Guinée, Guilcher, in Lauff, 1967).

- Sédiments d'origine fluviale : c'est le cas des cours d'eau à débit de crue élevé, comme la Loire (Berthois, 1955 ) et la Gironde (Latouche, 1971; Martin, 1971), ainsi que de tous les estuaires qui ont donné naissance à des deltas.

- Sédiments d'origine mixle : ce sont les cas intermédiaires, nombreux et variés, où les influences marines et fluviales sont en antagonisme. Germaneau (1968 à 1971) a décrit avec précision l'exemple de la Seine : les apports fluviaux se manifestent par de gros galets fossiles situés principalement à l'amont, de faibles quantités de sable en «suspension permanente» vers l'embouchure, et surtout de 70 à $80 \%$ de particules fines qui se déposent partout; les apports marins sont essentiellement sableux (diamètre supérieur à 100 microns) et sont surtout localisés dans la partie inférieure de l'estuaire, où ils sont apportés par les dérives littorales plutôt que par les courants de marée.

B. - LE DEVENIR DES SÉdIMENTS APPORTÉS AUX ESTUATRES PAR LES Fleuves fait également l'objet de controverses. Pour certains, le bouchon vaseux, qui contient un tonnage considérable de suspensions arrachées au fond, se déplace sans pratiquement jamais être expulsé, ce qui doit provoquer un envasement accéléré si l'apport fluvial est notable; pour d'autres, le bouchon est en partie éliminé et renouvelé à chaque cycle de marée. Une donnée intéressante résulte de la découverte, dans l'estuaire de la Loire (Berthois, 1970), d'une "lentille d'eau immobile» située contre le fond, entre le «coin salé » et l'eau fluviale : cette lentille s'oppose au charriage des matériaux grossiers, et ne permet que le passage des suspensions fines. Sa présence ou son absence, qui est conditionnée notamment par la pente moyenne du fond de l'estuaire, peut expliquer des situations multiples et changeantes. Ainsi Germaneau (1968 à 1971), dans la Seine, montre que les suspensions permanentes se déplacent vers l'aval et sont évacuées, cependant que les suspensions semi-permanentes se déplacent vers l'amont. D'une façon générale, le sable demeure à proximité de sa région d'origine (fleuve en amont, mer en aval) car, transitant près du fond, il rencontre des difficultés pour franchir les barrages de suspensions et d'eaux piégées. En revanche, les particules fines peuvent «sauter» ces barrages lorsqu'elles sont en suspension et, de ce fait, transitent plus facilement dans l'estuaire (Giresse, 1969; Germaneaul, 1968 à 1971).

\section{Évolution des estuaires au cours du temps}

Les estuaires sont rarement décrits dans les séries géologiques (ex. : Silurien d'Utica, U.S.A., Twenhofel, 1961). Cela peut tenir à la difficulté qu'il y a de les mettre en évidence, car la composition de leurs sédiments est très variable, comme on l'a vu. Mais ces zones mixtes, où s'affrontent la mer et les fleuves, sont aussi très instables, et on peut se demander si les estuaires sont facilement conservés.

Les estuaires actuels procèdent d'une évolution qui a débuté avec la fin de la dernière glaciation pleistocène (Würm) : le niveau de la mer était alors situé à proximité de la rupture de pente du plateau continental; les sédiments, apportés par les fleuves, étaient déversés directement sur le talus, vers les plaines abyssales, domnant des turbidites dans l'axe des canyons. Il n'y avait donc ni estuaires, ni marais côtiers.

Au début des temps post-glaciaires, il y a 10000 ans environ, la mer est remontée progressivement sur le plateau continental dont la pente est faible : les eaux fluviales se sont étalées sur ce glacis, abandonnant des sédiments dans de nombreux marais maritimes.

A la fin de la transgression post-glaciaire, la mer s'est stabilisée à un niveau voisin de l'actuel, au sommet du plateau continental, et a envahi la base des vallées fluviatiles : ainsi sont nés les estuaires que nous connaissons (Emery, in Lauff, 1967). A la transgression marine postglaciaire s'ajoute, pour Bourcart (1958), un mouvement d'abaissement đu précontinent, appelé flexure continentale.

Cette évolution passée explique l'abondance des estuaires actuels en regard des estuaires anciens, et il semble que nous sommes les témoins d'une époque privilégiée pour l'observation de ces zones originales, époque éphémère à l'échelle géologique. Ainsi, la côte des Etats-Unis présente-t-elle 80 à $90 \%$ de figures estuariennes sur la façade Atlantique, 10 à $20 \%$ sur la façade Pacifique.

L'évolution future des estuaires peut être de deux sortes. Si la mer se retire à nouveau, les alluvions seront érodées par suite de la recrudescence des apports fluviaux, et le cycle pourra recommencer (Russell, in Lauff, 1967). Si la mer continue de monter, elle ne fera qu'accélérer le processus actuel, qui tend à combler les estuaires, à partir de matériel d'origine marine, fluviale ou mixte. Les différentes étapes de ce comblement, aui sa poursuit actuellement à raison de $2 \mathrm{~m} / 1000$ ans en moyenne dans les régions humides (Rusnak, in Lauff, 1967), rappellent les termes de la classification des estuaires proposée par Glangeaud (1938) : vallée submergée (rias), estuaire en entonnoir (Loire), estuaire barré (Charente), estuaire à goulet (Gironde), estuaire lagunaire (Côte d'Ivoire), estuaire comblé (Grandes Landes).

Enfin, si le fleuve est important, le comblement de l'estuaire conduit à l'édification d'un delta et de lagunes, qui seront d'autant plus développés que les marées seront plus faibles (Mississippi, Rhône). Les différentes étapes qui mènent de l'estuaire au delta peuvent être reconstituées grâce à l'étude des structures sédimentaires livrées par les carottes (Reineck et De Vries Klein, in Lauff, 1967).

\section{Notions de sédimentologie appliquée}

Les estuaires sont des régions de grande importance en matière d'équipement, surtout lorsqu'ils constituent des voies de communication privilégiées. Il est donc nécessaire de bien connaitre la nature des sédiments qui occupent 
leur fond, et les études géotechniques sont particulièrement utiles à cet égard : tassement, rigidité, thixotropie des vases, etc.

D'autres renseignements précieux sont relatifs à la mobilité des sédiments estuariens, qui posent des problèmes fréquents, souvent inattendus, d'envasement ou d'ensablement. Parfois, la sédimentation contrarie la navigation, et c'est le cas des grandes dunes hydrauliques qui se déplacent dans les chenaux sous l'action des courants de marée. Un moyen, souvent utilisé, pour préserver les chenaux de la sédimentation est de les retrécir ou de rectifier leur parcours pour augmenter la vitesse des courants. Des conditions météorologiques exceptionnelles peuvent provoquer l'obstruction de ports estuariens, comme celui de Nantes, après un étiage déplaçant le bouchon vaseux très en amont, ou celui de Saint-Nazaire, après un déplacement latéral excessif de ce bouchon.

$\mathrm{Si}$ certains estuaires présentent manifestement des signes d'obstruction (Seine) ou d'érosion (Rance avant les travaux hydroélectriques), d'autres estuaires, parcourus par des chenaux navigables, paraissent stabilisés (Charente). Mais cet équilibre est très fragile, et les interventions humaines sont souvent catastrophiques : l'approfondissement d'un chenal, à plus forte raison son élargissement, peut provoquer un envasement massif, au cours du cycle de marée qui suit. Le rejet de dragages en zone estuarienne semble parfois responsable de la formation de «crème de vase», boue liquide qui envahit le fond des chenaux (in Martin, 1971).

Signalons enfin l'accumulation de rejets industriels dans les vases estuariennes, qui constituent des pièges à polluants d'autant plus redoutables qu'elles sont plus riches en argiles adsorbantes (montmorillonite...). Peu de mesures semblent avoir été faites dans ce domaine; on peut cependant citer, dans les sédiments de la Gironde (Martin, 1971), une concentration d'oligoéléments dix à cent fois supérieure à celle présentée par les sédiments normaux.

\section{Conclusion}

Malgré l'homogénéité texturale de ses dépôts, marqué par l'ubiquité de la vase, l'estuaire apparaît comme un milieu sédimentaire extrêmement varié. Cela se manifeste dans la répartition des sédiments sur les sections longitudinale et transversale de l'estuaire, dans la composition et l'origine des fractions sédimentaires, dans le mode de dépôt, dans la dynamique des suspensions, dans l'évolu- tion générale de ces régions au cours du temps. Le milieu estuarien est donc, en fait, hétérogène et discontinu. C'est pourquoi, dans la recherche de sa meilleure connaissance, qui est rendue urgente par les problèmes actuels d'environnement, les études sur modèles expérimentaux doivent être nécessairement complétées par des études précises du milieu naturel.

\section{Bibliographie sommaire}

Allen (G.P.), Castaing (P.), Feral (A.), Klingebiel (A.) et VigneAuX (M.). - Contribution à l'étude des faciès de comblement et interprétation paléogéographique de l'évolution des milieux sédimentaires récents et actuels de l'estuaire de la Gironde. Bull. Inst. Géol. Bass. Aquitaine, 8, p. 99-154. (1970).

Berthors (L.). - « La sédimentation dans l'estuaire de la Loire», Soc. Hydrotechn. France, fasc. roneo., 14 p. (1955).

Berthols (L.). - « Océanographie sédimentologique ». Centre Document. universit, Paris, 336 p. (1970).

Bourcart (J.). - «Problèmes de géologie sous-marine », Masson, coll. Evolut. des Sciences, 127 p. (1958).

Derruau (M.). - "Précis de géomorphologie ». Masson, Paris, 415 p. (1967).

Glangeaud (L). - Transport et sédimentation dans l'estuaire et à l'embouchure de la Gironde. Bull. Soc. Géol. France, (5), 8, p. 599-631. (1938).

Gullcher (A.). - «Morphologie littorale et sous-marine ». Presses Universit. France, coll. Orbis, 216 p. (1954).

LaufF (G.H.). — "Estuaries ». Public. 83 de l'Americ. Assoc. for the Advanc. of Science, 757 p. (1967).

Millor (G.). - «Géologie des argiles 》. Masson, Paris, 499 p. (1964).

OTtmann (F.). - «Introduction à la géologie marine et littorale 》. Masson, Paris, 259 p. (1965).

Ottmann (F.), Barbaroux (L.), Mahe (A.) et Moulin (G.). "Etude sédimentologique, géochimique et géotechnique des vases estuariennes (vasière de Mean, près St-Nazaire, estuaire de la Loire) 》. RCP 144, Nantes, Labor. Géologie marine, 11 p. -t annexe. (1970).

TWenhofel (W.H.). - «Treatise on sedimentation », Dover Public., 2 vol., 926 p. (1961).

Van Straaten (L.M.J.U.). - «Deltaïc and shallow marine deposits ». Elsevier, Amsterdam, Developm. in Sedimentology, 1, 464 p. (1964).

Thèses de doctorat ès-Sciences soutenues récemment en France : Allen (Gironde), 1972, Bordeaux; ChAmley (Rhône), 1971, Marseille; Germanenu (Seine), 1968 à 1971, La Rochelle. Giresse (Sienne), 1969, Caen; Latouche (Gironde), 1971, Bordeaux; Lecolde (Bandama, Côte-d'Ivoire), 1972, Nice; Martin (Gironde, Ems), 1971, Paris; Monnet (Bandama, Côte-d'Ivoire), 1972, Nice. 


\section{Discussion}

président : M. J. LaRras

M. le Président remercic M. Chamlex pour son intéressant exposé et poursuit :

Vous avez insisté sur le fait que le mot floculation désigne deux phénomènes complètement différents. Vous nous avez également rassurés : avant que je prenne ma retraite les estuaires n'auront pas tous disparu. Et, point vraiment intéressant, vous nous avez montré la force et la faiblesse d'un certain nombre d'affirmations ou d'hypothèses courantes. C'est de tout cela que je voudrais vous remercier, parce que certains disent : tout le monde sait ce qu'est la floculation qui est le phénomène principal ou qui est, au contraire, négligeable. D'autres, dans le même esprit, disent : tout le monde sait que les estuaires ne sont pas voués au comblement, d'autres que le phénomène va aller en s'aggravant, indépendamment de toute question de changement de nivcau de la mer.

Je voudrais ajouter à votre exposé quelques mots sur le phéno. mène des estuaires qui se déplacent latéralement; on en a un exemple au droit de Berck où le vent a déplacé l'estuaire vers le nord, pour autant qu'on peut le savoir, d'une bonne dizaine de kilomètres depuis les Romains; ceci n'a rien à voir avec les transports littoraux qui, évidemment, ont toujours tendance à créer une flèche sur un côté de l'estuaire, mais à l'action du vent qui remblaie progressivement la rive sud de l'estuaire au moyen de sable arraché des parties plus au sud de la côte par l'action éolienne. On a pu déterminer la position de l'estuaire depuis deux à trois siècles d'une façon très précise et l'on constate, ainsi, un cheminement latéral extrêmement curieux vers le nord.

Je tiens à vous féliciter à nouveau car bien que spécialiste de la mer Méditerranée, qui est une mer sans marée, dans laquelle le nombre d'estuaires est très faible et où l'on trouve plutôt des deltas, votre exposé constitue une très bonne introduction à nos débats en nous montrant ce qui est et ce qui n'est pas, ce qui est possible et ce qui ne l'est pas, ce qui est sûr et ce qui est, malgré tout, encore douteux.

J'ouvre la discussion pour ceux qui voudraient poser des questions à notre conférencier.

J'ai été très intéressé, dit $M$. LAPICQue, par votre remarque tendant à prouver que, si le niveau moyen de la mer monte, on doit tendre à récupérer des terres du fait du rôle bien connu des estuaires en matière de sédimentation. Il me semble que cet effet quelque peu paradoxal mérite d'être souligné..

Je pense, en effet, confirme M. CHAMLEx, que si le niveau de la mer monte à l'échelle des temps géologiques, nous gagnerons certainement des terres, parce que l'antagonisme hydrodynamique entre la mer et le fleuve va augmenter; il en résultera un dépôt de vase (marine ou fluviale) plus important. L'origine des estuaires et des deltas est précisément cette montée du niveau marin au cours des âges récents.

I1 m’a semblé comprendre, observe M. le Président, que vous attribuiez à la vase le rôle principal dans les estuaires; c'est vrai, mais pas dans tous les estuaires : certains estuaires africains sont essentiellement à base de sable.

11 est bien certain, confirme M. Mignior (Laboratoire Central d'Hydraulique de France) que le rôle du sable dans certains estuaires ne peut être négligé; en Loire, par exemple, M. BERTHOIs et les ingénieurs du Port de Nantes et Saint-Nazaire ont observé des apports de sable représentant environ le quart de l'apport total en sédiments.

Se référant aux expériences faites au L.C.H.F., M. Migkiotor n’est pas tout à fait d'accord avec les résultats présentés par M. ChamLey et tient à préciser qu'il suffit d'une faible quantité d'eau de mer pour obtenir, en eau calme, une floculation totale des particules de vases.

D'une façon générale, on constate que la vitesse de chute des flocons de vase augmente progressivement et rapidement lorsque la salinité des eaux passe de 0 à $3 \%$. Au-delà de cette valeur, et jusqu'à $10 \%$, laccroissement de la vitesse de chute est très faible ef l'on peut observer, dans certains cas, une légère diminution de la valeur de la vitesse maximale de chute lorsque l'on atteint des salinités de l'eau de mer à $30 \%$

De même, il est difficile d'aftirmer que les flocons de vases son toujours dispersés dans un courant et réduits à l'état de particules élémentaires. Des mesures, faites en nature par la Section d'Applications des Radio-éléments du C.E.A. tendraient à montrer le contraire. Ces expériences laissent présumer, en effet, que la vitesse de décantation des particules déplacées dans un courant d'eau pourrait être légèrement supérieure à celle des flocons en eau calme. I semblerait que, dans certains cas, la floculation est accélérée par la turbulence du courant, conduisant à la formation d'agrégats ayant des vitesses de chute supérieures à celles mesurées sur les flocons en eall calme. Il est probable que l'influence de la concentration en particules solides dans la suspension ne doit pas être négligeable et des expériences systématiques seraient nécessaires pour confirmer ces premiers résultats.

M. KÉRISEL (Port Autonome de Rouen) intervient en ces termes

M. CHAMLEY a cité certaines études qui tendraient à affirmer que l'alimentation en sable, par l'aval de l'estuaire de la Seine, se ferait principalement par transit littoral.

Ceci me surprend. Je pense, en effet pour ma part, que le transit littoral ouest-est n'amène dans l'estuaire de la Seine que quelques centaines de milliers de mètres cubes par an, alors que lo volume des sables provenant du large de l'estuaire, sous l'effet de la houle et du courant de flot, est de l'ordre de plusieurs millions de mètres cube par an. Ceci me paraî́ démontré par le volume des dragages effectués dans certains ports de la côte du Calvados qu ont un chenal nord-sud et par les constatations faites à l'occasion de la mise au point du grand modèle réduit à fonds mobiles de l'estuaire de la Seine.

J'ai tiré cette information du travail monumental de Germaneau sur l'estuaire de la Seine, mais je ne dispose d'aucune autre observation personnelle sur ce point, répond $M$. Chamley.

Après avoir remercié $M$. CHAMLEX et toutes les personnes aui ont animé la discussion, M. le Président clôt la discussion dar la remarque suivante :

Lintervention humaine joue un rôle considérable par la construction de barrages sur les rivières et par les extractions de matériaux pour la construction, tout le long de leur lit; ces dernières deviennent de plus en plus considérables et se chiffrent par millions de mètres cube chaque année.

M. le Président donne ensuite la parole successivement à M. Bonnefille et à M. Allen pour l'exposé de la communication qu'ils ont rédigé en collaboration. 